\title{
Etiquetas RFID Passivas e Aprendizagem de Máquina para Sistema de Localização indoor de Alta Precisão
}

\author{
Eduardo L. Gomes ${ }^{1,2}$, Mauro Fonseca ${ }^{1}$, Carlos R. Guerber ${ }^{1,2}$, Anelise Munaretto ${ }^{1}$ \\ ${ }^{1}$ Programa de Pós Graduação em Engenharia Elétrica e Informática Industrial (CPGEI) \\ Universidade Tecnológica Federal do Paraná (UTFPR) \\ Curitiba - PR - Brasil \\ ${ }^{2}$ Instituto Federal de Educação, Ciência e Tecnologia de Santa Catarina (IFSC) \\ Canoinhas - SC - Brasil \\ eduardo.gomescifsc.edu.br, maurofonsecalutfpr.edu.br
}

\begin{abstract}
Resumo. A localização de objetos em ambientes internos é uma tarefa desafiadora onde não há contato físico ou campo de visão e existe a presença de materiais reflexivos além do excesso de obstáculos. Para contornar tais problemas, nós propomos o uso da tecnologia RFID e métodos de aprendizagem de máquina para a construção de sistemas de localização em ambientes internos. As principais contribuições deste artigo são: um sistema de localização de alta precisão, modelagem específica para ambientes com prateleiras e o módulo de aprendizagem de máquina, o qual extrai e deriva estatisticamente características das leituras RFID. O resultado obtido com a implantação em um ambiente real foi de $100 \%$ de acurácia com o classificador Random Forest.
\end{abstract}

\section{Introdução}

Atualmente, os sistemas de localização estão presentes no cotidiano das pessoas devido aos benefícios que podem oferecer. Um sistema de localização pode ser definido como um conjunto de dispositivos, técnicas, algoritmos e aplicações que trabalham em sincronia para estimar as coordenadas absolutas ou relativas de uma pessoa ou objeto em um determinado ambiente previamente mapeado [Berz and Hessel 2015].

Em termos de localização outdoor, a tecnologia GPS (Global Positioning System) tornou-se o padrão quando se trata de localização. No entanto, a tecnologia GPS não é apropriada para ambientes internos, devido as limitações dos dispositivos atuais, como a falta de linha de visada entre os satélites e o receptor [Dinh-Van et al. 2017]. Desta forma, o foco de pesquisas associadas à localização em ambientes internos tem se baseado na busca por tecnologias apropriadas [Torres-Sospedra et al. 2015].

Devido a sua versatilidade e custo-benefício, muitas pesquisas usaram a tecnologia RFID (Radio Frequency IDentification) para propor sistemas de localização indoor. O RFID é um método de identificação automática que utiliza sinais de radiofrequência para identificar, rastrear e gerenciar objetos, animais ou pessoas sem qualquer contato físico ou campo de visão. Isso só é possível devido ao uso de etiquetas eletrônicas que refletem o sinal recebido de uma antena conectada a um leitor de RFID [R. Smith 2013]. Entretanto, algumas características frequentemente encontradas em ambientes internos aumentam a complexidade dos sistemas de localização que utilizam radiofrequência [Calderoni et al. 2015], como: 
1. A alta densidade de itens, onde é necessária uma margem de precisão muito alta, pois dependendo do ambiente os itens estão posicionados muito próximos uns dos outros;

2. O número de obstáculos, como paredes, móveis, equipamentos, plantas e outros;

3. A presença de materiais com superfície reflexiva, por exemplo metais e vidros.

Estas características são responsáveis por gerar interferências na propagação das ondas de radiofrequência. Os principais problemas gerados são [Westcott et al. 2011]:

1. Reflexão: O sinal de rádio pode ser refletido por alguns tipos de superfícies, devido à polarização do sinal elétrico nas ondas de rádio. Isso pode causar um desvio do sinal, fazendo com que ele não chegue ao destino esperado [Rappaport 2001];

2. Difração: A difração ocorre quando um sinal colide com uma superfície e se divide em dois ou mais sinais de rádio. Estas frações de sinais são refletidas para sentidos diferentes e com uma intensidade menor que o sinal original. Assim ocorre a atenuação de sinal e sua área de alcance diminui, o que pode impedir que o sinal chegue ao seu destino [Westcott et al. 2011];

3. Dispersão: A dispersão acontece quando um sinal colide com uma superfície refletiva que apresenta diferentes ângulos de reflexão, como em uma superfície áspera ou enrugada. Com isso, o sinal se divide em outros sinais menores e de menos intensidade, que ao se colidirem com outros sinais presentes no ambiente, se transformam em ruídos [Rappaport 2001].

Estas interferências tornam o cálculo da localização complexa devido à falta de uniformização dos valores recebidos pelo leitor RFID, especialmente a Indicação da Força do Sinal Recebido (RSSI) que é a característica predominantemente utilizada para medir a distância entre os leitores RFID e as etiquetas eletrônicas [Ma et al. 2018]. Essa falta de uniformização dos valores em um sistema que usa técnicas simples de triangulação ou trilateração faz com que um objeto seja identificado em movimento, mesmo permanecendo imóvel.

Neste artigo, com o objetivo de contornar tais problemas, nós propomos uma arquitetura de localização para ambientes internos utilizando etiquetas RFID passivas. A arquitetura integra os componentes de infraestrutura, negócios e comunicação. Também, extrai estatisticamente características dos dados brutos recebidos do leitor RFID que contribuem para que os algoritmos classificadores de aprendizagem de máquina identifiquem a posição onde estão localizadas as etiquetas.

Este artigo está organizado da seguinte forma: A seção 2 demonstra os trabalhos relacionados e as principais contribuições do presente trabalho. A seção 3 apresenta em detalhes como todos os módulos e processos da arquitetura de localização proposta foram construídos. A seção 4 apresenta os resultados obtidos na aplicação do sistema usando dados de treinamento e em um ambiente real, e a seção 5 apresenta as conclusões e sugestões para trabalhos futuros.

\section{Trabalhos Relacionados e Principais Contribuições}

A utilização da tecnologia RFID para criar sistemas de localização e posicionamento indoor é amplamente utilizada em vários estudos na literatura. Especialmente em razão dos benefícios que pode fornecer como: segurança contra furtos, agilidade de identificação 
de pessoas, localização de produtos, inventário de estoque, posicionamento e navegação de robôs, além de diversas outras aplicações [Torres-Sospedra et al. 2015]. Conforme pudemos levantar, uma única solução multiuso que atenda a qualquer necessidade em aplicações de posicionamento interno ainda não existe. De fato, os sistemas propostos na literatura baseiam-se em diversas tecnologias e modelos adaptados, projetados para atender às exigências de cada contexto específico.

Para o presente artigo, focamos nos modelos de sistema para localização indoor que utilizam como base o atributo RSSI, pois é calculado automaticamente nos leitores RFID que estão disponíveis no mercado, não necessitando de equipamentos especiais ou fabricantes específicos.

[Hori et al. 2008] propuseram um método de estimativa de localização de etiquetas RFID usando uma abordagem probabilística (Bayesiana) onde robôs móveis com leitores RFID estimam as posições das etiquetas fixas com o objetivo de locomover-se em um ambiente interno. Outra abordagem foi usada por [Nakamori et al. 2012] para navegação de robôs. A proposta apresentada por eles foi denominada como sistema de navegação de movimento contínuo, que usou dois intervalos de comunicação: intervalos longos e intervalos curtos e os alternou adequadamente estimando a posição das etiquetas RFID que também estavam fixas no ambiente. Já [Ting et al. 2011] estudaram a viabilidade de usar etiquetas RFID passivas para detecção de posicionamento de objetos e rastreamento de movimento em tempo real. Os resultados do experimento mostraram que a acuracidade do sistema de posicionamento RFID passivo foi satisfatória considerando uma precisão de 1 metro. [Akre et al. 2014] demonstraram uma proposta para a localização de etiquetas passivas usando a abordagem de aprendizado do ambiente. Neste método utilizaram uma função agregada do RSSI para todos as potências possíveis de transmissão dos leitores RFID e com base nessas medidas definiram uma assinatura da localização para utilizar no algoritmo K-Nearest Neighbor (KNN). [Steele et al. 2019] criaram um sistema utilizando as técnicas Angle of Arrival e Time Difference of Arrival para identificar o deslocamento de pacientes em unidades de saúde. Algumas medidas importantes para análise dos médicos foram extraídas do deslocamento como: distância percorrida, velocidade média, tempo na zona de interesse (quarto) e tempo sem atividade.

O atributo RSSI, devido à sua importância, é fortemente investigado em várias pesquisas de sistemas de localização que utilizam radiofrequência. [Zhang et al. 2015] investigaram o uso de estatísticas de RSSI para identificar a posição de uma etiqueta RFID passiva em relação a um leitor. Além disso, concluíram que quanto maior a distância das antenas maior a interferência da orientação da etiqueta em relação às antenas. No estudo de [Hatem et al. 2018], foram avaliados os erros na estimativa de distâncias para localização em ambientes internos com o objetivo de criar um modelo de perda de caminho que foi empregado para minimizar os erros de localização. [Ma et al. 2018] em sua pesquisa não criaram um modelo de localização, mas sim um modelo de detecção automática de leituras de falsos positivos em um leitor RFID. Para isso, realizaram a derivação do RSSI em conjunto com algoritmos de aprendizagem de máquina e concluíram que o RSSI é fortemente influenciado pelo ambiente, como os reflexos de múltiplos caminhos de sinais e obstrução que são muito comuns em ambientes internos. [Calderoni et al. 2015] propuseram um sistema de localização indoor projetado para funcionar em cenários ruidosos, onde as transmissões podem ser interferidas por outros dis- 
positivos eletrônicos ou paredes protegidas como é o caso de um hospital. O objetivo foi detectar a sala em que pacientes se encontravam e em $98 \%$ dos casos o sistema localizou a sala correta ou uma de suas adjacências. [Wang et al. 2019] apresentaram um sistema de localização indoor com o objetivo de reduzir ao máximo o processo de implantação, onde somente a posição dos leitores RFID devem ser conhecidas. Utilizaram o atributo RSSI de etiquetas ativas e os métodos de Newton-search e Kalmann Filter como técnicas de localização. Os resultados foram obtidos em tempo real utilizando simulador.

Mesmo em sistemas de localização indoor que não utilizam RFID, mas têm abordagem utilizando radiofrequência como $\mathrm{Wi}-\mathrm{Fi}$, é notável a preocupação com a sensibilidade do ambiente para o RSSI. [Torres-Sospedra et al. 2015] realizaram uma pesquisa com o objetivo de identificar qual a melhor função de distância a ser aplicada em algoritmos que usam a abordagem do algoritmo KNN para minimizar a sensibilidade do RSSI. Eles concluíram que é muito difícil aplicar algoritmos de propagação de sinal ao posicionamento sem fio devido à natureza severa da propagação de sinal. [Dinh-Van et al. 2017] propuseram um método de WiFi-fingerprint para substituir o comportamento do GPS em ambientes internos. A principal contribuição foi usar uma técnica de suavização de dados brutos com o método de classificação (rede neural) para tratar a intensidade do sinal Wi-Fi ruidoso. [Sabr and Belton 2019] propuseram uma solução de identificação e localização que utilizou uma combinação de duas tecnologias: reconhecimento facial e Wi-Fi utilizando trilateração do atributo RSSI. Fizeram testes em ambiente controlado e concluíram que a precisão do RSSI foi diretamente influenciado pela quantidade de equipamentos no ambiente.

No artigo [Gomes et al. 2017] nós apresentamos uma arquitetura de localização indoor que obteve $100 \%$ de acerto na localização de livros em uma biblioteca. A arquitetura utilizava a etiqueta passiva como uma classe alvo na construção dos classificadores e isso se tornou uma vulnerabilidade na implantação em ambientes de grande escala, pois a complexidade da construção do classificador e tempo de processamento foram proporcionais à quantidade de classes. Para evitar estes problemas, no presente trabalho nós propomos a divisão das etiquetas em macro regiões que são definidas através da clusterização hierárquica. Inicialmente é realizada uma classificação que indica qual macro-região pertence o objeto e após há uma segunda classificação considerando somente as etiquetas da macro-região. Desta maneira reduzimos exponencialmente a quantidade de classes na construção dos classificadores.

A Tabela 1 apresenta uma comparação dos modelos de localização propostos nos trabalhos relacionados. Nós identificamos algumas lacunas que são motivação para nossa pesquisa. É comum em ambientes internos, que para sua organização, os objetos sejam dispostos em prateleiras, como por exemplo: bibliotecas, supermercados, farmácias, armazéns de distribuição e estocagem. Esta característica não é abordada especificamente em nenhum dos trabalhos relacionados. Outra característica importante é a precisão, pois devido a alta densidade de objetos nestes ambientes se faz necessário que estejam dispostos muito próximos uns dos outros. Dentre os trabalhos relacionados a maior precisão encontrada foi de 20 centímetros, isto demonstra que objetos menores de 20 centímetros não podem ser posicionados lado a lado inutilizando espaços no ambiente e podendo até inviabilizar a implantação do sistema. 
Tabela 1. Comparação dos Trabalhos Relacionados

\begin{tabular}{|l|l|l|l|l|l|}
\hline Pesquisa & Alvo & Cenário & Sensor & Precisão & Movimentação \\
\hline Este Artigo & Livros & Prateleiras & Etiqueta Passiva & $5 \mathrm{~cm}$ & $\checkmark$ \\
\hline [Hori et al. 2008] & Robôs & Simulado & Etiqueta Passiva & $100 \mathrm{~cm}$ & \\
\hline [Nakamori et al. 2012] & Robôs & Simulado & Etiqueta Passiva & $22 \mathrm{~cm}$ & \\
\hline [Ting et al. 2011] & Etiqueta & Controlado & Etiqueta Passiva & $100 \mathrm{~cm}$ & $\checkmark$ \\
\hline [Akre et al. 2014] & Etiqueta & Controlado & Etiqueta Passiva & $20 \mathrm{~cm}$ & $\checkmark$ \\
\hline [Zhang et al. 2015] & Etiqueta & Controlado & Etiqueta Passiva & $40 \mathrm{~cm}$ & \\
\hline [Calderoni et al. 2015] & Pessoa & Salas & Etiqueta Ativa & Acima de $10 \mathrm{mt}$ & \\
\hline [Torres-Sospedra et al. 2015] & Fingerprint & UJI indoorLoc db & WiFi-Fingerpint & $170 \mathrm{~cm}$ & \\
\hline [Dinh-Van et al. 2017] & Veículo & Estacionamento & WiFi-Fingerpint & $10 \mathrm{mt}$ & \\
\hline [Hatem et al. 2018] & Etiqueta & Controlado & Etiqueta Ativa & $50 \mathrm{~cm}$ & $\checkmark$ \\
\hline [Wang et al. 2019] & Pessoa & Simulado & Etiqueta Ativa & $100 \mathrm{~cm}$ & \\
\hline [Sabr and Belton 2019] & Pessoa & Controlado & Wi-Fi & $100 \mathrm{~cm}$ & $\checkmark$ \\
\hline [Steele et al. 2019] & Pessoa & Unidade de Saúde & Etiqueta Ativa & $100 \mathrm{~cm}$ & $\checkmark$ \\
\hline
\end{tabular}

\subsection{Principais Contribuições}

Diante das lacunas ainda não abordadas nos trabalhos relacionados e do principal problema para os sistemas de localização indoor que é a interferência do ambiente na propagação de ondas de radiofrequência, o presente artigo apresenta uma arquitetura de localização indoor usando a tecnologia RFID com etiquetas passivas. As principais contribuições são:

1. Alta precisão (cinco centímetros);

2. Modelagem específica para ambientes com prateleiras;

3. Módulo de aprendizagem de máquina, o qual processa os dados recebidos pelo leitor RFID, extrai e deriva estatisticamente características, contribuindo para a redução dos erros de localização devido ao problema de propagação de ondas de radiofrequência.

A seção seguinte (seção 3) apresenta em detalhes como todos os módulos e processos da arquitetura de localização proposta neste trabalho foram construídos.

\section{Sistema de Localização indoor}

Geralmente, um sistema RFID é composto de componentes de infraestrutura e componentes de negócio. Os componentes de infraestrutura são as etiquetas, leitores, cabos, conectores e antenas. Os componentes de negócios são as regras do negócios, aplicações, softwares de gerenciamento e o middleware que realiza a comunicação entre componentes de infraestrutura e componentes de negócio [R. Smith 2013]. A Figura 1 apresenta o modelo conceitual do sistema de localização indoor, o mesmo é dividido em dois módulos. O Módulo de Leitura que faz parte do componente de infraestrutura e o Módulo de Aprendizagem de Máquina que faz parte dos componentes de negócio.

Com o objetivo de validar o sistema em um ambiente real, uma biblioteca foi utilizada como estudo de caso. Nós escolhemos este ambiente, pois tipicamente os objetos (livros) estão posicionados lado a lado em prateleiras. As seções 3.1 e 3.2 apresentam em detalhes como cada um dos módulos do sistema foi construído.

\subsection{Módulo de Leitura}

São os componentes básicos de infraestrutura de um sistema RFID (etiquetas, antenas e leitores). O leitor geralmente está conectado a um computador com capacidade suficiente para processar os dados recebidos das etiquetas. Para a preparação do ambiente de 


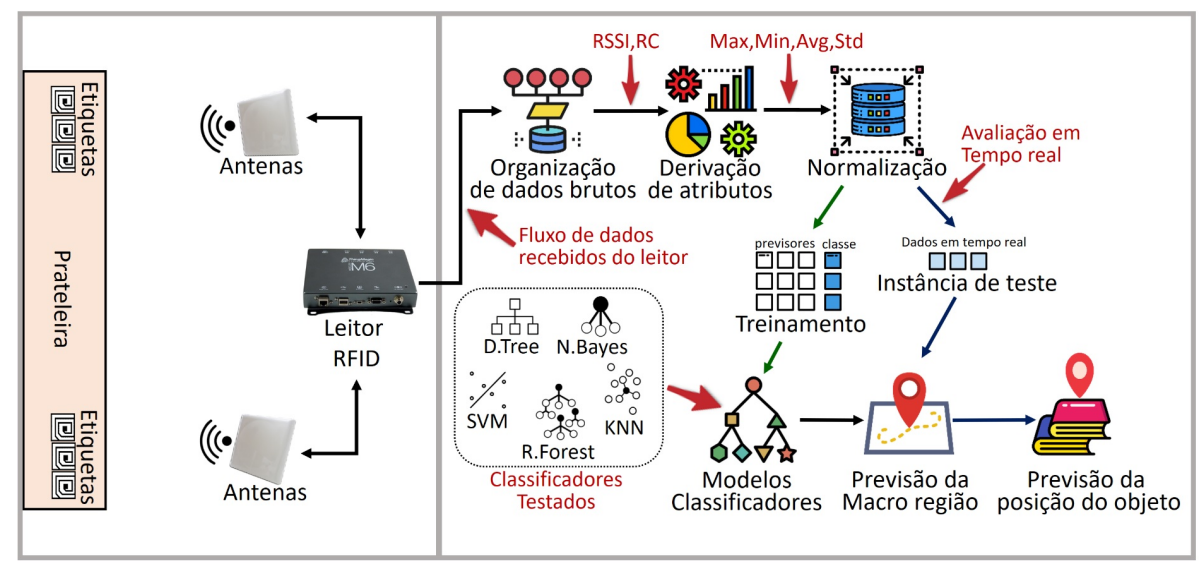

(a) Módulo de Leitura

(b) Módulo Aprendizagem Máquina

Figura 1. Modelo Conceitual do Sistema de Localização indoor

implementação, nós utilizamos quatro antenas UHF mono-estáticas que operam na faixa de frequência de 902 a $928 \mathrm{MHz}$ com ganho de $6 \mathrm{dBi}$ (ganho de antena isotrópica). O equipamento que usamos para realizar as leituras foi o ThingMagic Mercury 6, que é um leitor RFID UHF de alto desempenho, suportando até quatro antenas monoestáticas, entradas e saídas digitais e conexão Wi-Fi. Ambos os equipamentos são comerciais e podem ser comprados por qualquer pessoa em empresas especializadas na comercialização de equipamentos RFID. Nós fixamos vinte e oito etiquetas verticalmente na capa dos livros os quais foram posicionados lado a lado nas prateleiras da biblioteca. A distância entre as antenas e etiquetas foi de $(\mathbf{1 2 0} \mathbf{~} \mathbf{~ m})$ e a distância entre o grupo de antenas (210 cm). O tempo de interrogação definido foi de $\mathbf{5}$ segundos, este é o tempo que o leitor interroga as etiquetas e organiza as informações de entrada em uma coleção de dados formada pelos campos timestamp, RSSI, TagID, Read Count e Antenna (Figura 2(a)). Para definir este tempo nós identificamos o limite mínimo no qual todas as etiquetas foram identificadas pelo menos uma vez em uma antena. Para realizarmos a coleta de dados os livros com etiquetas foram colocadas em posições conhecidas. Realizamos 100 leituras em cada etiqueta alvo, medindo o RSSI e o RC de cada uma das 4 antenas, totalizando 2.800 leituras. A Figura 2(a) apresenta os atributos e parte do fluxo de dados enviados pelo leitor e encaminhados para o Módulo Aprendizagem de Máquina (seção 3.2).

\subsection{Módulo Aprendizagem de Máquina}

Conforme apresentado na Figura 1, o Módulo Aprendizagem de Máquina é responsável por receber os dados brutos do Módulo de Leitura, organizar o fluxo de dados, derivar os atributos (RSSI e RC), normalizar os dados, construir os classificadores e realizar a predição das posições das etiquetas. Para a construção deste módulo nós utilizamos a linguagem de programação Python com a biblioteca Scikit-learn [Pedregosa et al. 2011]. Nas seções 3.2.1, 3.2.2, 3.2.3 e 3.2.4 nós detalhamos como cada processo se comporta.

\subsubsection{Organização de Dados Brutos}

Processo que recebe o fluxo de dados do Módulo de Leitura e organiza os valores RSSI e RC de cada uma das antenas. A Figura 2(a) exibe uma amostra de como os dados são 
(a) Fluxo de dados enviado pelo leitor

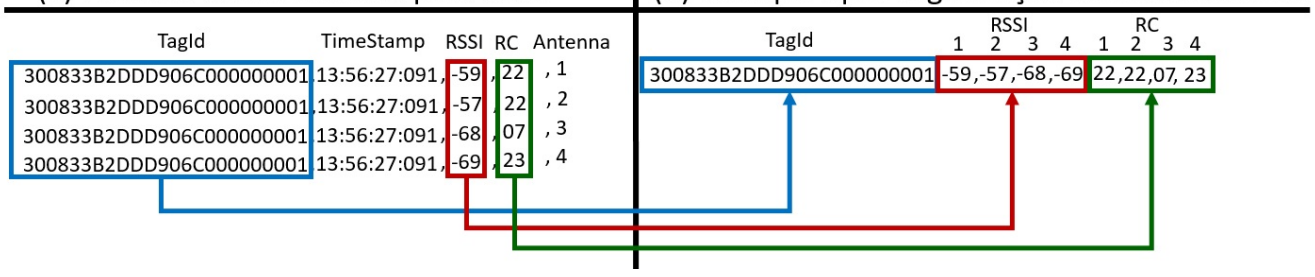

Figura 2. Processo Organização de Dados Brutos
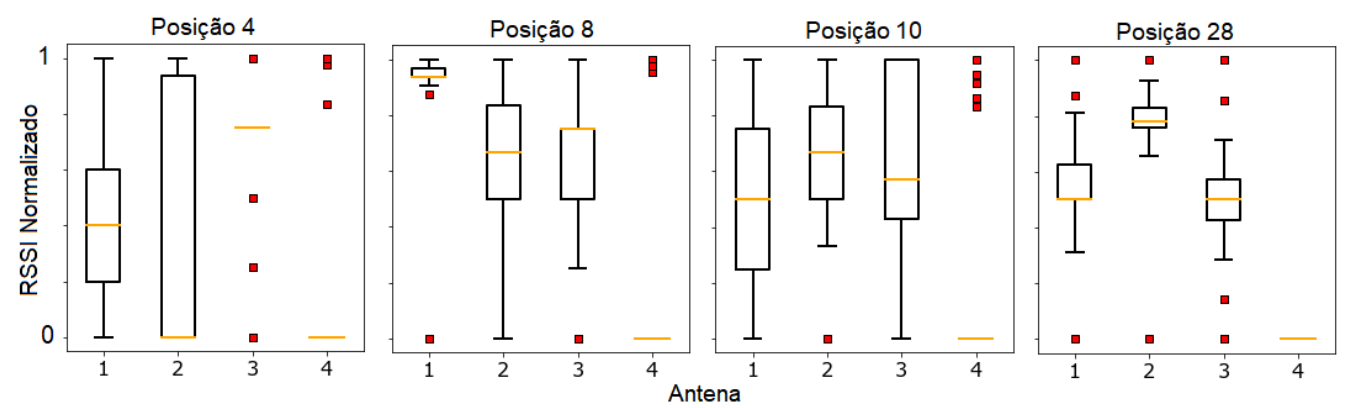

Figura 3. Análise de outliers do atributo RSSI

recebidos do leitor RFID e a Figura 2(b) mostra como os dados são organizados após este processo. Assim como em outros trabalhos da literatura (seção 2), ao analisarmos os dados nós identificamos uma variação significativa do RSSI. A Figura 3 apresenta um boxplot com uma amostra das leituras realizadas em quatro posições, demonstrando a presença de muitos outliers. Além disso, o intervalo médio entre os valores mínimo e máximo do RSSI é de $94.7 \%$, o que dificulta a precisão na construção dos classificadores de aprendizagem de máquina.

\subsubsection{Derivação de Atributos}

Segundo [Spiegel and Stephens 2015], entre as técnicas de estatística descritiva existentes, quando há um grupo de dados, os primeiros a serem utilizados são as medidas de sumarização. Para os dados quantitativos que é o caso do presente trabalho, existem medidas de posição (média, moda, mediana, percentil, quartis), medidas de dispersão (desvio padrão, amplitude, intervalo interquartil, variância e coeficiente de variação) e medidas de forma (skewness, kurtosis). No sistema proposto no presente trabalho, nós usamos uma técnica de medidas de posição (média) e uma técnica de medidas de dispersão (desvio padrão) para a derivação dos atributos RSS e RC.

O processo Derivação de Atributos agrupa o número de tuplas recebidas do processo Organização de Dados para identificar os valores mínimo e máximo e calcula as médias e o desvio padrão de cada atributo (RSSI e RC) para as quatro antenas. A Tabela 2 demonstra a estrutura da coleção de dados após a finalização desse processo. A quantidade de atributos aumenta para quarenta, dez atributos para cada antena. 
Tabela 2. Derivação de Atributos. Onde $n$ representa o número da antena.

\begin{tabular}{|l|c|l|}
\hline Atributo & Tipo de dado & Descrição \\
\hline rssi_antena $n$ & float & RSSI antena $n$ \\
\hline rc_antena $n$ & integer & Quantidade de leituras da etiqueta na antena $n$ \\
\hline avg_rssi_antena $n$ & float & Média do RSSI \\
\hline avg_rc_antena $n$ & float & Média do Read Count \\
\hline min_rssi_antena $n$ & float & Valor mínimo do RSSI \\
\hline min_rc_antena $n$ & float & Valor mínimo do Read Count \\
\hline max_rssi_antena $n$ & float & Valor máximo do RSSI \\
\hline max_rc_antena $n$ & float & Valor máximo do Read Count \\
\hline stddev_rssi_antena $n$ & float & Desvio padrão do RSSI \\
\hline stddev_rc_antena $n$ & float & Desvio padrão do Read Count \\
\hline
\end{tabular}

\subsubsection{Normalização}

A base de dados de treinamento (Tabela 2) possui essencialmente atributos numéricos e de diferentes magnitudes. Para evitar que estas diferentes magnitudes interfiram no comportamento de alguns classificadores nós normalizamos os valores de cada atributo. Para normalizar os dados nós utilizamos a classe StandardScaler da biblioteca Scikitlearn.

\subsubsection{Classificadores e Predição}

Dentre as técnicas existentes de aprendizagem de máquina nós utilizamos a classificação para identificar a posição das etiquetas nas prateleiras. A classificação de dados consiste em duas fases, a fase de treinamento e a fase de predição [Mitchell 1997]. Na fase de treinamento os algoritmos de aprendizado de máquina constroem um classificador baseado no conjunto de treinamento formado por tuplas de dados (atributos) e seus rótulos correspondentes (classes). A tupla é composta por um vetor de características n-dimensional representado por $X=\left(x_{1}, x_{2}, \ldots x_{n}\right)$. No modelo apresentado neste artigo, quarenta características são extraídas das leituras RFID, $\log 0 n=40$ e a classe alvo são as posições das etiquetas no ambiente representadas pelo número da posição (1 a 28).

Em grande escala a utilização da etiqueta individual como uma classe alvo poderá gerar problemas de classificação, pois a complexidade da construção do classificador e tempo de processamento são proporcionais à quantidade de classes. Para evitar estes problemas nós dividimos as etiquetas em macro regiões, onde há inicialmente uma classificação que indica qual macro-região pertence o objeto e após há uma segunda classificação considerando somente as etiquetas da macro-região. Para definir a quantidade de macro-regiões e também definir qual macro-região pertence cada posição, nós utilizamos a técnica de aprendizagem de máquina de clusterização hierárquica. A clusterização hierárquica, é um algoritmo que começa com todos as classes atribuídas a um cluster próprio. Em seguida, dois clusters mais próximos são mesclados no mesmo cluster. Finalmente, termina quando há apenas um único cluster, construindo uma hierarquia entre eles [Bonaccorso 2017]. Os resultados do agrupamento hierárquico podem ser visualizados no gráfico dendrograma. A melhor escolha do número de clusters é o número de linhas verticais no dendrograma cortadas por uma linha horizontal que pode atravessar verticalmente a distância máxima sem intersectar um cluster. No ambiente de implementação apresentado neste artigo o melhor número de clusters encontrado foi 3 


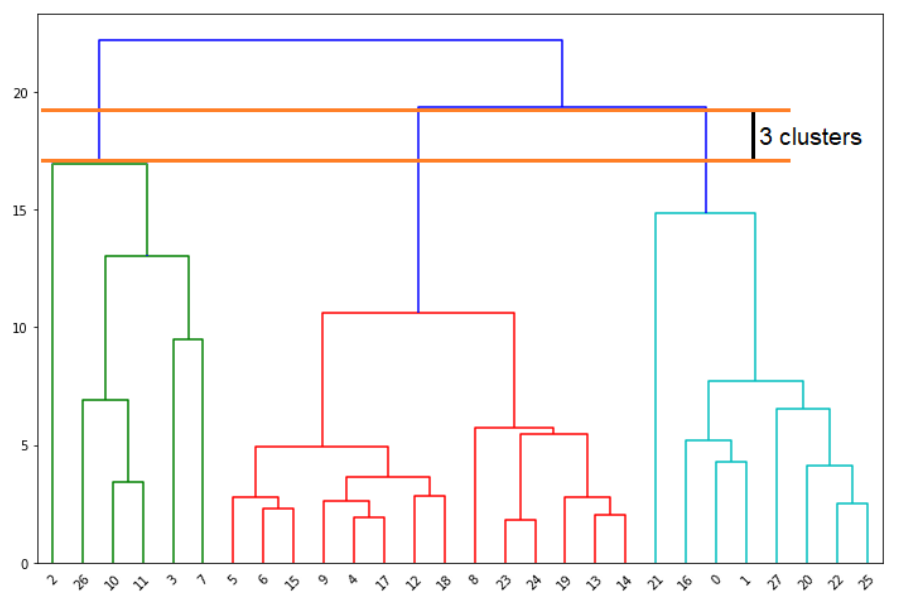

Figura 4. Dendrograma para definição das macro-regiões

clusters. A Figura 4 apresenta o dendrograma gerado com a base de dados de treinamento, como foram definidas as macro-regiões e suas respectivas posições.

Para criar os classificadores nós testamos cinco algoritmos de aprendizagem de máquina comumente estudados na literatura: Decision Tree(DT) [Han et al. 2012], Random Forest(RF) [Breiman 2001], Naive Bayes(NB) [Aggarwal 2014], K-Nearest Neighbor(KNN)[Aggarwal 2014] e Support Vector Machine(SVM) [Ma et al. 2018].

Para construir o classificador DT nós utilizamos a classe DecisionTreeClassifier com parâmetro criterion igual a Gini. O índice de Gini mede a impureza do conjunto de dados e é utilizado para encontrar o critério de divisão na árvore. Para construir o classificador RF nós utilizamos a classe RandomForestClassifier com parâmetro criterion igual a Gini e parâmetro n-estimators igual a 20. O parâmetro n-estimators indica a quantidade de classificadores DT que serão gerados na RF. Para construir o classificador NB nós utilizamos a classe GaussianNB. Para construir o classificador KNN nós utilizamos a classe KNeighborsClassifier com parâmetro n_neighbors $=5$ (número de vizinhos), metric $=$ minkowski e $p=2$ (parâmetro $p=2$ indica para o classificador utilizar a distância euclidiana). E para construir o classificador SVM nós utilizamos a classe $S V C$ com parâmetro kernel $=r b f$ e $C=2$. A seção 4 apresenta os resultados dos testes realizados.

\section{Avaliação Experimental}

Nossa estratégia de análise de desempenho focou em duas etapas. Inicialmente realizamos testes para identificar o quanto o processo Derivação de Atributos influencia nos resultados. Para este teste utilizamos os dados da base de treinamento e métricas apresentadas na seção 4.1. Na segunda etapa, realizamos testes para avaliar o desempenho do sistema em tempo real, realizando a troca de posições dos livros. Para este etapa, usamos os dados capturados em tempo real apresentados na seção 4.2.

\subsection{Avaliação dos dados de treinamento}

A avaliação da base de dados de treinamento é importante para verificar se as amostras dos dados são representativas o suficiente para que os classificadores obtenham uma acurácia 


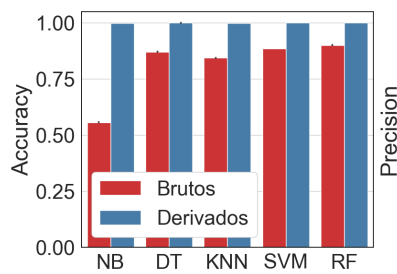

(a)

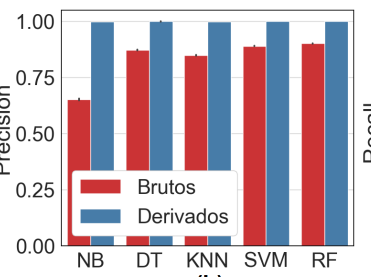

(b)

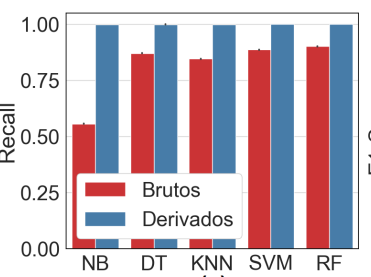

(c)

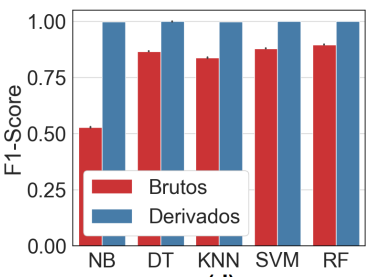

(d)

Figura 5. Atributos Brutos x Atributos Derivados.

aceitável no ambiente de implantação. Para realizar os testes, nós utilizamos a classe StratifiedKFold da biblioteca Scikit-learn e testamos a base de dados de treinamento 30 vezes para cada classificador usando a técnica de validação cruzada [Kohavi 1995] com 10 folds. Em cada um dos 30 testes, variamos a semente geradora de 1 a 30, constituindo uma estimativa média das métricas Accuracy, Precision, Recall e F1 Score de cada classificador. Accuracy é a medida de desempenho mais intuitiva e é simplesmente uma razão de observação corretamente prevista para o total de observações e é calculada pela equação

$$
\text { Acurracy }=\frac{T_{p}+T_{n}}{T_{p}+F_{p}+F_{n}+T_{n}}
$$

onde $T_{p}$ são os verdadeiros positivos, $T_{n}$ são os verdadeiros negativos, $F_{p}$ são os falsos positivos e $F_{n}$ são os falsos negativos. A Precision é a razão de observações positivas preditas corretamente para o total de observações positivas preditas e é definida pela equação

$$
\text { Precision }=\frac{T_{p}}{T_{p}+F_{p}}
$$

Recall ou Sensitivity é a razão de observações positivas corretamente previstas para todas as observações na classe investigada e calculada pela equação

$$
\text { Recall }=\frac{T_{p}}{T_{p}+F_{n}}
$$

F1 Score é a média ponderada de Precision e Recall. Portanto, essa métrica leva em conta tanto os falsos positivos quanto os falsos negativos, conforme mostrado na equação

$$
F_{1}=2 \times \frac{\text { Recall } \times \text { Precision }}{\text { Recall }+ \text { Precision }}
$$

A Figura 5 apresenta os resultados obtidos pelos classificadores usando somente os atributos RSSI e RC que chamamos de Atributos Brutos, em comparação com os atributos RSSI e RC acompanhados dos atributos derivados Mínimo, Máximo, Média e Desvio Padrão que chamamos de Atributos Derivados. Percebemos que em todos os classificadores houve um ganho significativo no resultado de todas as métricas. Estes resultados demonstram que a técnica de derivação de atributos proposta nesta pesquisa contribui significativamente para a melhora do desempenho de todas as técnicas de classificação investigadas.

A Tabela 3 apresenta especificamente os resultados médios obtidos pelos classificadores utilizando os Atributos Derivados. Percebemos que há uma semelhança nos valores de todas as métricas de cada classificador. Isso significa que os dados de treinamento 
Tabela 3. Resultados usando atributos derivados

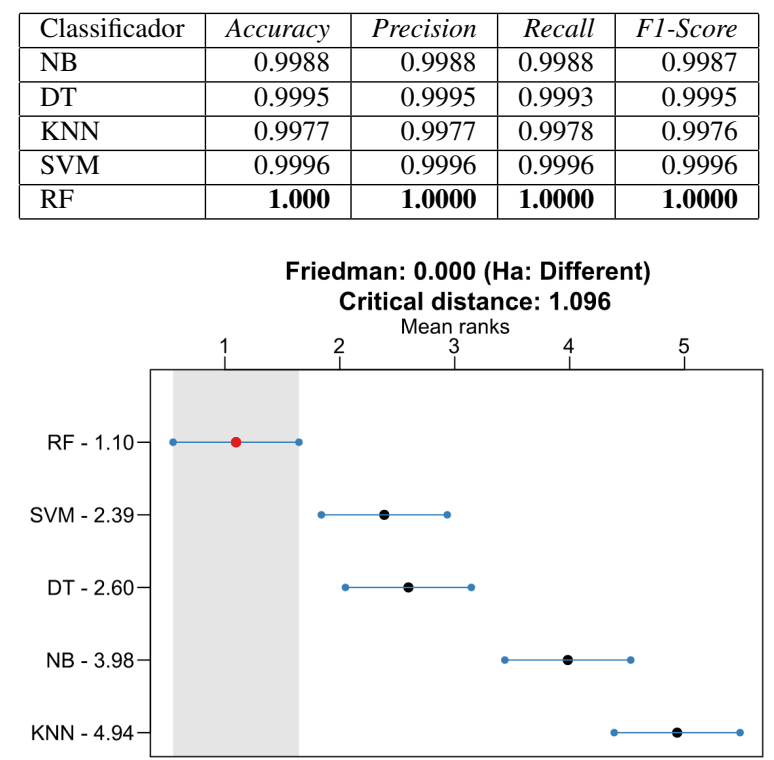

Figura 6. Testes de Friedman e Nemenyi.

possuem uma distribuição uniforme para todas as classes, o que permite reconhecer que a métrica Accuracy apresenta resultados confiáveis. Também percebemos que o classificador RF obteve um melhor resultado. Para determinar se houve ou não diferença estatisticamente significativa entre as médias dos resultados dos classificadores, realizamos os testes de Friedman [Kvam and Vidakovic 2007] e Nemenyi para a métrica Accuracy. A Figura 6 mostra que o teste de Friedman identificou que há uma diferença significativa no ranking dos classificadores. A distância crítica (CD) foi 1.096, que indica que a diferença entre o ranking dos classificadores deve ser maior que esse valor para caracterizar uma distância estatisticamente significativa. Portanto podemos concluir que o classificador RF obteve melhor desempenho neste experimento, pois não há outro classificador com resultado no mesmo intervalo.

\subsection{Resultados em tempo real}

Para avaliação em tempo real usamos a base de dados de treinamento para construir os modelos classificadores e o fluxo de dados recebidos em tempo real como instância de teste (Figura 1(b)).

Com o objetivo de identificar o desempenho do sistema proposto em tempo real, trocamos todos os 28 livros de posição e realizamos novas leituras. A Figura 7(a) apresenta os resultados obtidos após a troca de todos os livros. Podemos identificar que na primeira apuração de resultados houve uma redução na taxa de acerto nos classificadores NB, KNN, SVM e DT. Essa redução foi esperada porque cada livro possui diferente espessura e tamanho, afetando a posição original. Mesmo assim, o classificador DT e o classificador SVM obtiveram desempenho muito semelhante à posição original, $98.57 \%$, $98.90 \%$ respectivamente, e o classificador RF se manteve com $100 \%$ de acerto. Na segunda apuração de resultados, houve um aumento na taxa de acertos, pois adicionamos as leituras da primeira apuração de resultados à base de dados de treinamento que contribuíram para a melhoria do desempenho dos classificadores. Na terceira apuração de 

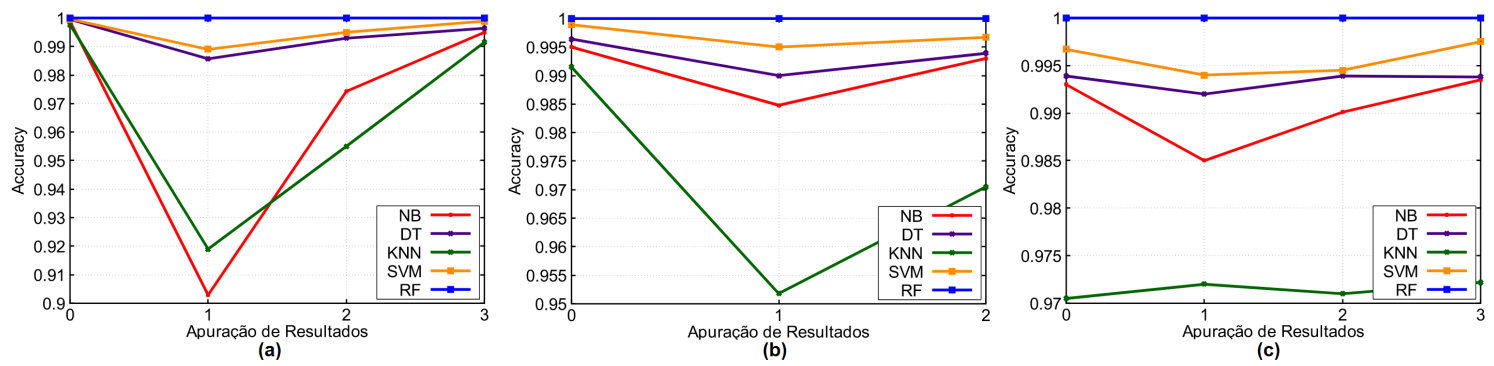

Figura 7. Evolução da acurácia após a movimentação de todos livros (a), após a movimentação de dois livros (b) e após a movimentação de seis livros (c).

resultados, observamos que a taxa de acerto foi similar à posição original em todos os classificadores.

Após a terceira apuração de resultados em que todos os classificadores obtiveram desempenho semelhante em relação aos dados de treinamento, a nossa investigação se concentrou na troca de apenas dois livros para analisarmos o comportamento do sistema nessa situação. A Figura 7(b) mostra o resultado e neste caso, o classificador RF novamente se manteve em $100 \%$ e o sistema precisou de duas apurações de resultados para obter a acurácia semelhante nos outros três melhores classificadores(SVM, DT, e NB).

Realizamos novos testes em tempo real realizando a troca de posições de seis livros. A Figura 7(c) mostra a evolução da acurácia e neste caso, o sistema precisou de uma apuração de resultados para obter $100 \%$ no classificador RF, duas apurações para obter precisão semelhante no classificador SVM e DT e três para o classificador NB.

\section{Conclusões}

Sistemas de localização em ambientes fechados são amplamente estudados devido aos diversos benefícios que podem proporcionar como: controle de processos, inventário de estoque, rastreamento, navegação e antifurto. A tecnologia RFID possui uma grande versatilidade e utilizar etiquetas RFID passivas para criar sistemas de localização indoor contribui para a redução dos custos de implantação, pois além de possuir um preço menor em relação às etiquetas ativas, a tendência mundial é que todos os itens já possuam etiquetas desde a fabricação, percorrendo toda a cadeia de suprimentos com a mesma etiqueta. Entretanto, as interferências nas ondas de radiofrequência dificultam a obtenção de resultados satisfatórios em termos de acurácia, principalmente quando usada em ambientes com materiais reflexivos e excesso de obstáculos, frequentemente encontrados em ambientes internos.

Neste trabalho, nós apresentamos um sistema utilizando RFID e aprendizagem de máquina para localização indoor especificamente para ambientes que os objetos são posicionados em prateleiras, como por exemplo: bibliotecas, supermercados, farmácias, armazéns de distribuição e estocagem. Como resultado experimental em ambiente real onde havia prateleiras de metal e outros materiais reflexivos, a implementação do sistema apresentou uma acurácia de 100\% (classificador RF) após a movimentação de posicionamento de todas as etiquetas alvo. Para melhor generalização do modelo proposto, em trabalhos futuros é necessário implementar a arquitetura em outros ambientes.

O processo que merece destaque é o Derivação de Atributos, uma vez que as 
técnicas de derivação estatísticas dos atributos contribuíram significativamente para a acurácia. Além disso, esta técnica de derivação de atributos pode ser usada em outras aplicações de aprendizagem de máquina que possuam poucos atributos em sua base de dados de treinamento, independentemente do uso de sistemas RFID.

Apesar do bom desempenho do sistema e de sua adequação em relação aos objetivos iniciais, alguns aspectos importantes merecem investigações adicionais e servem como motivação para trabalhos futuros. No processo Derivação de Atributos, outras técnicas estatísticas podem ser usadas, como moda, mediana, percentil, quartis, amplitude, intervalo interquartil, variância, coeficiente de variação, skewness e kurtosis. No Módulo Aprendizagem de Máquina, outras técnicas de classificação podem ser implementadas como: Bagging, Boosting, Deep Learning, Drift Detection, Massive Online Analysis, etc. Outro trabalho poderia propor um sistema utilizando a abordagem de aprendizagem de máquina não supervisionada. Neste caso as posições das etiquetas seriam definidas pelo próprio sistema, reduzindo o tempo de implantação.

\section{Referências}

Aggarwal, C. C. (2014). Data Classification: Algorithms and Applications. Chapman \& Hall/CRC, 1st edition.

Akre, J.-M., Zhang, X., Baey, S., Kervella, B., Fladenmuller, A., Zancanaro, M., and Fonseca, M. (2014). Accurate 2-D localization of RFID tags using antenna transmission power control. 2014 IFIP Wireless Days (WD), pages 1-6.

Berz, E. L. and Hessel, F. P. (2015). Sistema híbrido de localização indoor baseado em rfid e análise visual. Faculdade de Informática, PUCRS. Porto Alegre-RS.

Bonaccorso, G. (2017). Machine Learning Algorithms: A Reference Guide to Popular Algorithms for Data Science and Machine Learning. Packt Publishing.

Breiman, L. (2001). Random forests. Mach. Learn., 45(1):5-32.

Calderoni, L., Ferrara, M., Franco, A., and Maio, D. (2015). Indoor localization in a hospital environment using RF classifiers. Expert Systems with Applications.

Dinh-Van, N., Nashashibi, F., Thanh-Huong, N., and Castelli, E. (2017). Indoor Intelligent Vehicle localization using WiFi received signal strength indicator. 2017 IEEE MTT-S International Conference on Microwaves for Intelligent Mobility.

Gomes, E. L., Fonseca, M., Munaretto, A., and Guerber, C. R. (2017). Arquitetura RFMiner - Uma solução para localização indoor. XXII Workshop de Gerência e Operação de Redes e Serviços(WGRS), pages 111-122.

Han, J., Kamber, M., and Pei, J. (2012). Data mining concepts and techniques, third edition. Morgan Kaufmann Publishers, Waltham, Mass.

Hatem, E., El-Hassan, B., Laheurte, J.-M., Abou-Chakra, S., Colin, E., and Marechal, C. (2018). Study the estimated distance error in indoor localization using uhf-rfid. 2018 IEEE Middle East and North Africa Communications Conference (MENACOMM).

Hori, T., Wada, T., Ota, Y., Uchitomi, N., Mutsuura, K., and Okada, H. (2008). A multisensing-range method for position estimation of passive RFID tags. 4th IEEE WiMob 2008, pages 208-213. 
Kohavi, R. (1995). A study of cross-validation and bootstrap for accuracy estimation and model selection. In 14th International Joint Conference on Artificial Intelligence, San Francisco, CA, USA. Morgan Kaufmann Publishers Inc.

Kvam, P. and Vidakovic, B. (2007). Nonparametric statistics with applications to science and engineering. 123.

Ma, H., Wang, Y., and Wang, K. (2018). Automatic detection of false positive RFID readings using machine learning algorithms. Expert Systems with Applications.

Mitchell, T. M. (1997). Machine Learning. McGraw-Hill, Inc., New York, NY, USA.

Nakamori, E., Tsukuda, D., Fujimoto, M., Oda, Y., Wada, T., Okada, H., and Mutsuura, K. (2012). A new indoor position estimation method of RFID tags for continuous moving navigation systems. 2012 International Conference on Indoor Positioning and Indoor Navigation, IPIN 2012 - Conference Proceedings, (November).

Pedregosa, F., Varoquaux, G., Gramfort, A., Michel, V., Thirion, B., Grisel, O., Blondel, M., Prettenhofer, P., Weiss, R., Dubourg, V., Vanderplas, J., Passos, A., Cournapeau, D., Brucher, M., Perrot, M., and Duchesnay, E. (2011). Scikit-learn: Machine learning in Python. Journal of Machine Learning Research, 12:2825-2830.

R. Smith, J. (2013). Wirelessly Powered Sensor Networks and Computational RFID.

Rappaport, T. (2001). Wireless Communications: Principles and Practice. Prentice Hall PTR, Upper Saddle River, NJ, USA, 2nd edition.

Sabr, O. and Belton, J. (2019). Identifying and Tracking Individuals in a Smart Indoor Environment. IEEE-Fifth International Engineering Conference on Developments in Civil \& Computer Engineering Applications.

Spiegel, M. R. and Stephens, L. J. (2015). Estatística: Coleção Schaum. Bookman Companhia Editora Ltda., Porto Alegre, RS, Brasil, 4rd edition.

Steele, A. M., Bopp, M. M., Rock, L., Boppvagov, M. M., Taylor, T. S., Sullivan, D. H., and Motivation, A. (2019). Patient Activity Monitoring Based on Real-Time Location Data. 2019 IEEE Int. Conf. on Bioinformatics and Biomedicine.

Ting, S. L., Kwok, S. K., Tsang, A. H. C. A., and Ho, G. G. T. S. (2011). The Study on Using Passive RFID Tags for Indoor Positioning. International Journal of ..., (1).

Torres-Sospedra, J., Montoliu, R., Trilles, S., Belmonte, Ó., and Huerta, J. (2015). Comprehensive analysis of distance and similarity measures for Wi-Fi fingerprinting indoor positioning systems. Expert Systems with Applications, 42(23):9263-9278.

Wang, J., Dhanapal, R. K., Ramakrishnan, P., Balasingam, B., Souza, T., and Maev, R. (2019). Active RFID Based Indoor Localization. IEEE-22nd International Conference on Information Fusion.

Westcott, D. A., Coleman, D. D., Miller, B., and Mackenzie, P. (2011). CWAP Certified Wireless Analysis Professional Official Study Guide. SYBEX Inc., CA, USA.

Zhang, X. b., Akre, J.-M. b., Baey, S. b., Fladenmuller, A. b., Kervella, B. b., Zancanaro, M. b. c., and Fonseca, M. (2015). Towards localization of RFID tags based on experimental analysis of RSSI. IFIP Wireless Days, (January). 